\title{
Testing the vestibular-ocular reflexes: abnormalities of the otolith contribution in patients with neuro-otological disease
}

\author{
HILARY BARRATT, ADOLFO M BRONSTEIN, MICHAEL A GRESTY \\ From the M.R.C. Neuro-Otology Unit, National Hospital for Nervous Diseases, Queen Square, London, UK
}

SUMMARY Conventional vestibular rotation testing with the head centred on the axis stimulates the semicircular canals evoking compensatory eye movements. If the head is placed forwards of the axis in an eccentric position the otoliths are also stimulated by a tangential linear acceleration acting laterally to the skull. In normal subjects the additional otolithic stimulus evokes compensatory eye movements with a higher gain than with head centred, particularly for high frequency $(>0 \cdot 1 \mathrm{~Hz})$ stimuli. The responses with head centred and eccentric in various patients with known/suspected neuro-otological abnormalities have been compared. Patients with vestibular neurinectomies who have asymmetrical head centred responses showed greater asymmetry with head eccentric at higher stimulus frequencies. Some patients with cerebellar lesions showed abnormally enhanced or depressed and asymmetrical responses with head eccentric in comparison with head centred responses, which could be normal. The enhancing effects could be specific to low frequency stimuli. All patients who showed abnormal responses with head eccentric also had positional nystagmus provoked by the gravity acceleration vector when the head was tilted laterally. The direction of the positional nystagmus with respect to the gravity vector was not necessarily the same as the direction of the effect on eye movements of lateral acceleration during eccentric oscillation. Patients with benign paroxysmal vertigo or chronic linear vertigo in whom otolithic abnormalities are suspected were not found to have abnormal responses with head eccentric. We conclude that this method of testing may be useful in elucidating pathophysiology but is not a decisive clinical test for the presence of disordered otolith function.

The otolith organs of the labyrinth transduce linear accelerations of the skull. Their physiology remains obscure for several reasons. There is the initial technical difficulty of constructing linear acceleration devices suitable for delivering test stimuli. Further problems arise because, according to Relativity Theory, gravity and acceleration are identical which means that the otolith organ itself cannot distinguish between reorientations of the gravity vector caused by head tilt and imposed linear accelerations. The am-

Address for reprint requests: Dr MA Gresty, National Hospital for Nervous Diseases, Queen Sq, London WEIN 3BG, UK.

Received 9 September 1986. Accepted 23 October 1986 biguous, otolithic afferent signal must be interpreted with respect to accompanying canal, visual and proprioceptive input in order to make a correct assessment of head motion and tilt and thereby to initiate appropriate reflexes and actions. This means that appropriate investigations of otolith function must be concerned with the complex interactions of the various senses and sensory motor systems. ${ }^{1}$

In spite of these problems, there is a need for tests of normal and abnormal otolith function, for it is clear that certain patients with neuro-otological disease show abnormalities which are presumably otolithic. Examples of symptomatic abnormalities of otolith function are misjudgement of true earth vertical or false sensations of lateropulsion and tilt. ${ }^{2}$ Accepted clinical signs suggestive of otolithic abnormalities are tilted body posture (particularly of the 
head), ${ }^{34}$ positional nystagmus of the central type* 56 and comitant torsion and skew deviation of the eyes. ${ }^{478}$

One way of investigating otolith function in humans is to study "otolith-ocular compensatory" eye movements. During fixation on a stationary target, the head may be moved either actively or passively and the line of sight automatically remains more or less aligned with the target direction so that vision is preserved. The mechanisms responsible for this visual stabilisation are the "compensatory" or "dolls" eye movements. Compensatory eye movements derive (1) from the semicircular canals of the labyrinth which provide a vestibular ocular reflex (canal-VOR) which compensates for angular head displacements; (2) from the smooth pursuit reflex which effects tracking of apparent target motion; (3) from the optokinetic reflex which stabilises the eyes with respect to large areas of the visual world and (4) possibly, from stimulation of the otoliths which could produce eye movements compensating for linear displacements of the skull. Previous investigators have sought to evoke eye movements by stimulating the otoliths with low frequency $(0.5 \mathrm{~Hz}$ and less) linear oscillation of the head, or by rotating the head about a tilted axis so that they are stimulated by the changing direction of the gravity vector. ${ }^{9}$ This form of otolith stimulation does not appear to evoke eye movements of significant velocity (of the order of $7 \% \mathrm{~s}$ maximum for $1 \mathrm{~g}$ stimulus). In contrast, we have demonstrated that high velocity otolith-related eye movements can be evoked by combined linear and angular motion. ${ }^{1011}$ The technique is to subtract the eye movement caused by angular motion alone from the eye movement caused by the angular motion combined with a linear motion. The difference between the two is an amount of eye movement attributable to the linear component of motion, and thus probably due to otolith stimulation. It is possible that the combined linear and angular head movement, which is the common occurrence during natural head movement, provides a contextual combination of canal and otolith signals which can be appropriately interpreted (as discussed above).

\footnotetext{
*Note on "positional nystagmus": definitions and mechanisms.

Positional nystagmus is a nystagmus which is provoked or modulated in slow phase velocity by static tilt of the head with respect to the gravity vector and occurs with disease of the central nervous system, the vestibular end organ or nerve. In "ageotropic positional nystagmus" the nystagmus fast phase or "beats" are in the opposite direction to the earth; the slow phase is towards the earth. In "geotropic positional nystagmus", the nystagmus fast phase or "beats" are in the direction of the earth; the slow phase is away from the earth. If the head is tilted sideways to horizontal with one ear down, gravity effects a stimulus to the otoliths which is the same as an acceleration upwards in the direction of the upper ear. If the head were actually moving then one might expect a compensatory slow phase eye movement to be made in the downwards direction; for example, head accelerating upwards to the left ear, eyes compensate by moving rightwards producing ageotropic nystagmus. Normal subjects do not have a reflex eye movement when tilted for the brain correctly interprets that the head is tilted and not accelerating. Ageotropic nystagmus in patients may be evidence of the "release" of some form of compensatory eye movement. Geotropic nystagmus is in the wrong direction to be compensatory.
}

The purpose of the present study was to assess the applicability of the technique in evaluating vestibularoculomotor disorders in a sample of patients with neuro-otological disease.

\section{Methods}

The experimental technique has been described extensively elsewhere. ${ }^{1011}$ In brief, the patient is seated upright, with head and body restraints, on a turntable which rotates about a vertical axis. The head may be centred on the axis to produce purely angular acceleratory stimuli which activate the semicircular canals and evoke lateral eye movements. Alternatively, the head may be offset eccentrically from the axis, in this case thrust $30 \mathrm{~cm}$ forwards (fig 1). Oscillation in the eccentric position produces the same angular stimulation and, in addition, involves a linear tangential acceleration vector which acts laterally on the skull to stimulate the otoliths. The motion stimuli used are relatively unpredictable "enveloped sinewaves" (fig 2) with constant peak angular velocity of $60^{\circ} / \mathrm{s}$ and centred on frequencies of $0.02,0 \cdot 1,0.5$ and $1.2 \mathrm{~Hz}$. The linear acceleratory stimuli at these frequencies are $0.004,0.02,0.1$ and $0.24 \mathrm{~g}$ respectively $(\mathrm{g}$ is the gravitational unit; $1 g=9.81 \mathrm{~m} / \mathrm{s}^{2}$ ). Each stimulus is delivered twice, one commencing with rightwards rotation, the other with leftwards rotation. Overall stimulus presentation is randomised except for the lowest frequency stimulus which is presented last because it is soporific. Eye movements are recorded using direct-coupled electrooculography. Testing is performed in darkness and the subject may be given no instructions other than encouragement to be alert or instructed to actively imagine fixating on earth fixed targets during the test runs. Instructions for imaginary fixation may considerably enhance vestibular responses; however, they are not used for patient testing be-

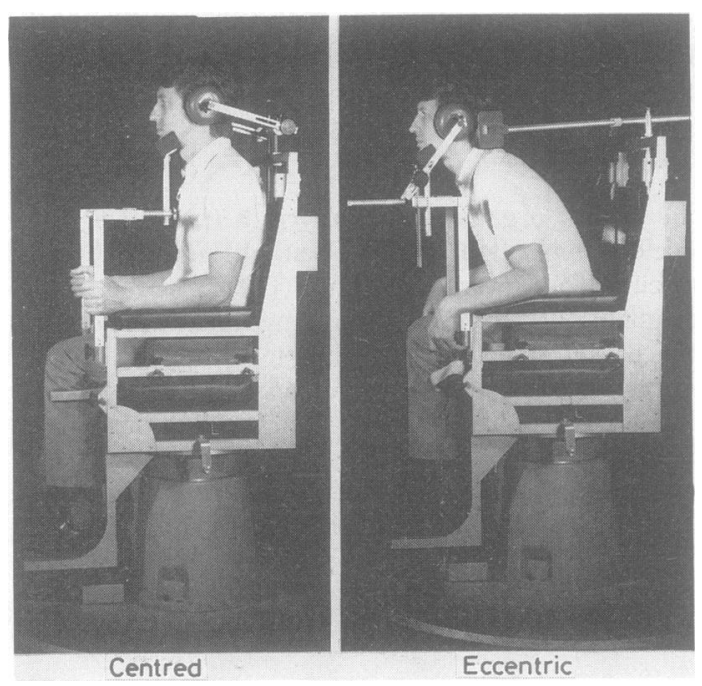

Fig 1 Experimental apparatus showing a subject seated in the conventional head centred position for rotational testing and seated with head $30 \mathrm{~cm}$ eccentric. 
(a) Head centre

Head $30 \mathrm{~cm}$ eccentric
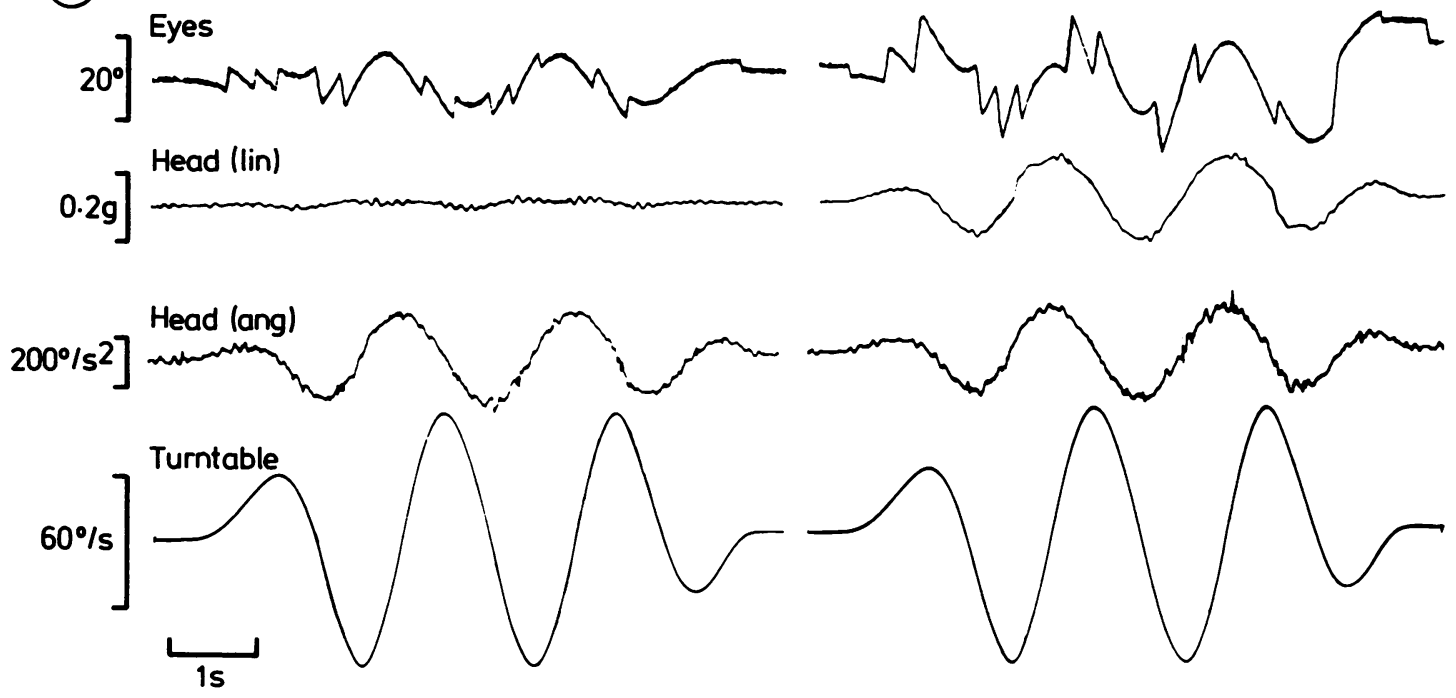

(b)
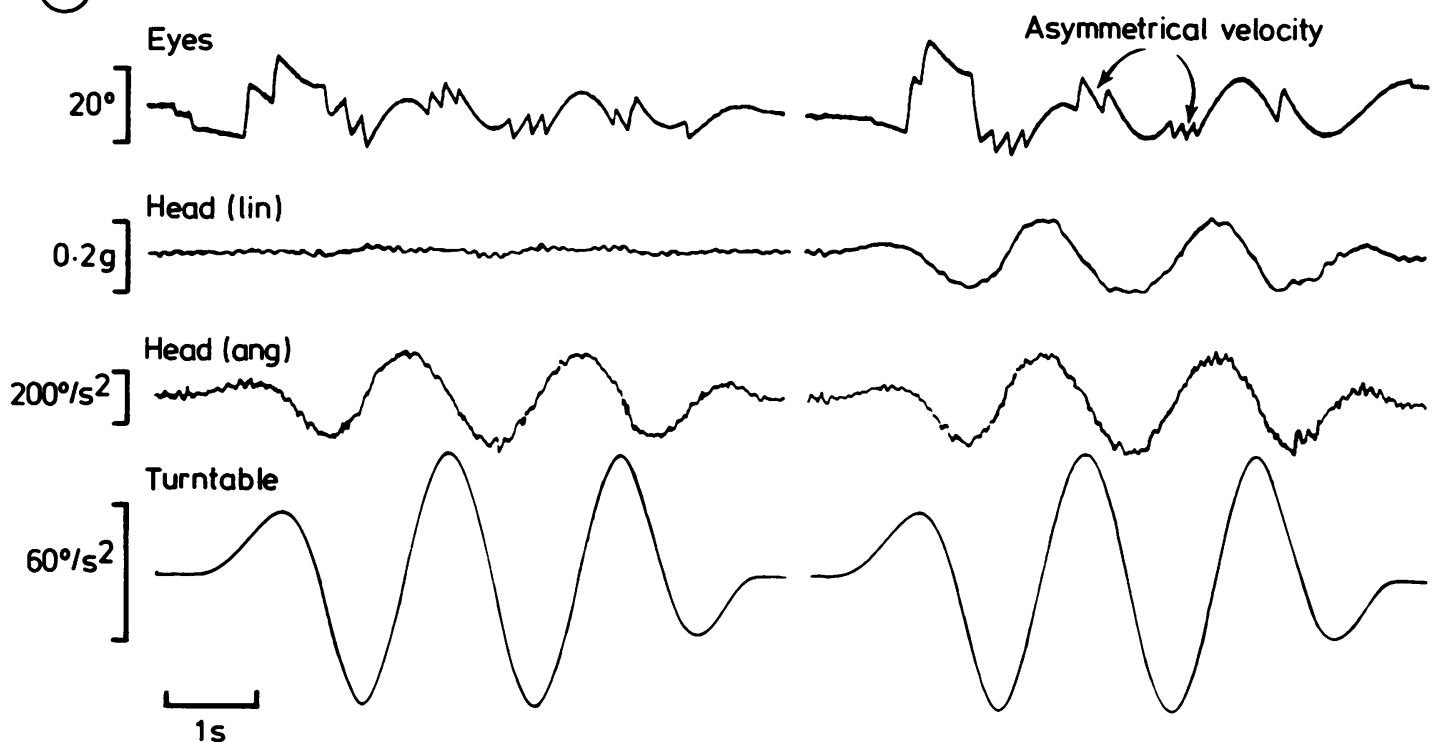

Fig 2(a) Raw data records of a normal subject's responses to centred and eccentric oscillation at $0.5 \mathrm{~Hz}$. (b) Raw data records of the responses of patient B11526. Head (lin): tangential linear acceleration acting laterally on the head. Head (ang) angular acceleration of the head.

cause we have encountered difficulties of comprehension in some patients.

In order to characterise the data of the patients presented in this study, measurements were taken of peak rightwards and leftwards slow phase eye velocity attained during the central, undistorted parts of the stimulus waveform. Because it was found that patients may show considerable rightwards/leftwards asymmetry, the measurements were separately averaged for rightwards and leftwards movements and expressed as velocity ratios (ie mean peak eye velocity rightwards/peak turntable velocity leftwards). Normal subjects showed negligible asymmetries so rightwards 

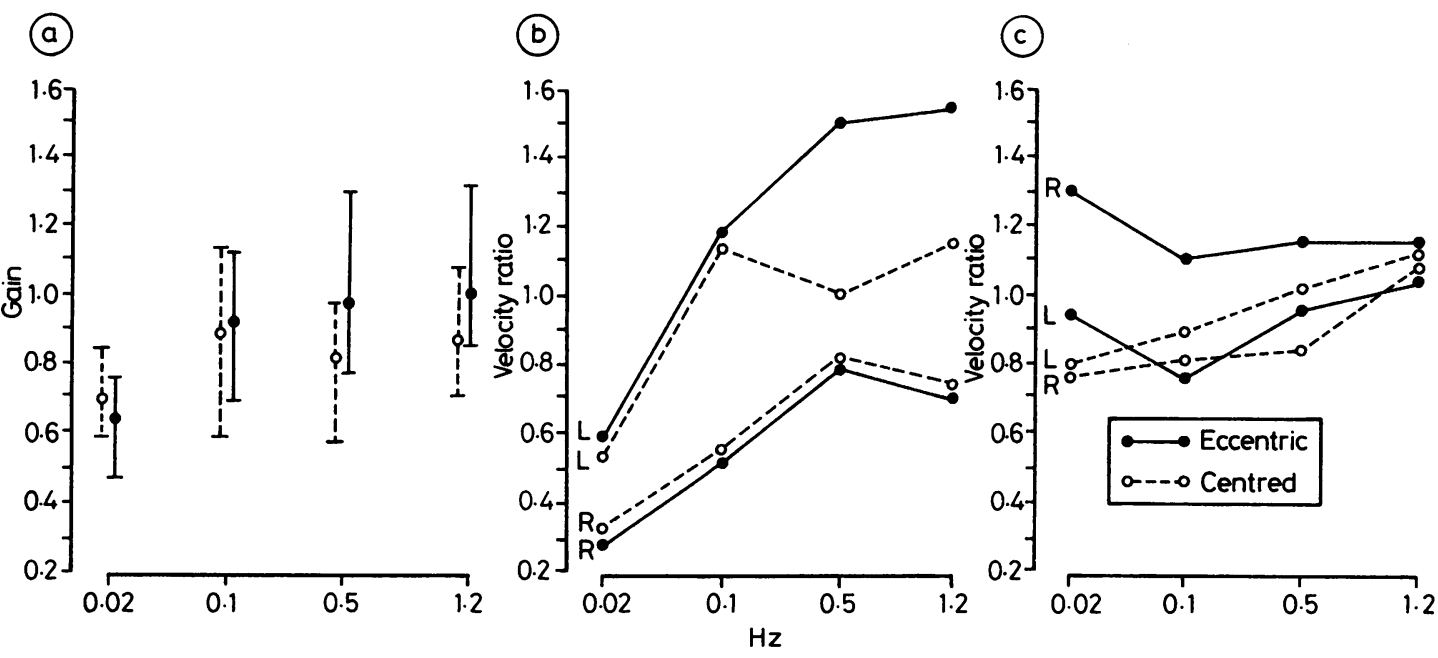

Fig 3(a) Normal data of gain against frequency showing means and ranges. Rightwards and leftwards responses grouped together. In the present discussion, patients responses are only identified as abnormal if they lie outside the normal range. (b) Patient B45846. Velocity ratios of peak eye velocity rightwards and leftwards/peak turntable velocity leftwards and rightwards respectively. (c) Patient B11526. Velocity ratios of peak eye velocity rightwards and leftwards/peak turntable velocity leftwards and rightwards respectively. $R=$ rightwards slow phase eye movements in response to leftwards rotation. $L=$ leftwards slow phase eye movements in response to rightwards rotation.

and leftwards movements were averaged together and expressed as gain (mean peak eye velocity/turntable velocity). Phase of response was measured as the relationship between the turning point of the slow phase eye movement with respect to zero turntable velocity. Phase measurements for $1 \cdot 2$ $\mathrm{Hz}$ condition may be up to $5^{\circ}$ in error. Phase estimates are more reliable for lower stimulus frequencies.

\section{Results}

Normal data have been presented in detail elsewhere. ${ }^{11}$ Normal subjects showed a significant increase in gain of slow phase eye movements in the eccentric position as compared with the head centred position at frequencies of 0.5 and $1.2 \mathrm{~Hz}$. There was also a trend towards elevation of gain at $0 \cdot 1 \mathrm{~Hz}$. For both centred and eccentric attitudes, the peak velocity of slow phase eye movement was in phase with the peak velocity of the stimulus at $0 \cdot 1 \mathrm{~Hz}$ and above. At $0.02 \mathrm{~Hz}$ the phase of the response was $20^{\circ}$ to $30^{\circ}$ in advance of the stimulus. An example of normal raw data records is presented in fig $2 \mathrm{a}$. Gain data for the normal subjects is presented in fig 3a. We attribute the elevation of gain in the head eccentric attitude to an otolithic contribution to the vestibular ocular reflex. However, it is not clear how the otolith signal controls eye movements. One possibility is of a direct otolith-ocular reflex similar to canal reflexes. Alternatively the otolith signal may be used to adjust the gain control of canal reflexes. ${ }^{11}$
Certain patients have been selected to illustrate test results because they exhibited various combinations of enhanced/decreased responses with head eccentric and geotropic/ageotropic positional nystagmus. In addition, most had normal canal-vestibular-ocular reflexes on conventional, head centred rotation tests. (1) Absence of positional nystagmus. Failure to enhance the VOR with lateral acceleration. Case example: B52832. Female aged 59 years who had suffered three episodes of vertigo over the previous two years. She was found to have bidirectional gaze paretic and rebound nystagmus in the lateral plane and almost absent smooth pursuit. Plantars were extensor. Magnetic resonance imaging showed appearances compatible with demyelination. Conventional rotational (with head centred) and caloric tests showed symmetrical hypoactive vestibular responses. There was a complete failure to evoke enhanced responses when tested with head eccentric.

(2) Positional nystagmus beating ageotropically with enhancement of the VOR by lateral acceleration (eye movement provoked by gravity in the same direction as eye movement provoked by acceleration). Case example: B45846. Female aged 45 years who was examined one year after the removal of a left sided acoustic neuroma. The significant vestibular findings were a total left sided canal paresis with spontaneous, right beating dark nystagmus which enhanced on positioning with the left ear down. On head centred oscil- 
lation the slow phase eye movements evoked by head leftwards stimuli were at the low limit of normal range at all frequencies. Responses to rightwards stimuli were all of consistently higher gain and within normal limits. With head eccentric the responses to rightwards stimuli were elevated beyond the normal range, at high frequencies. Responses to leftwards stimuli were not different from the responses to head centred leftwards stimuli (fig $3 b$ ). Response phase data for leftwards (L) and rightwards (R) slow phase eye movements were as follows:

$\begin{array}{ccccc} & 1.2 \mathrm{~Hz} & 0.5 \mathrm{~Hz} & 0.1 \mathrm{~Hz} & 0.02 \mathrm{~Hz} \\ \text { Head centre } & 0^{\circ} \mathrm{R}, 11^{\circ} \mathrm{L} & 2^{\circ} \mathrm{R}, 10^{\circ} \mathrm{L} & 3^{\circ} \mathrm{R}, 8^{\circ} \mathrm{L} & 16^{\circ} \mathrm{R}, 27^{\circ} \mathrm{L}\end{array}$

Head eccentric $0^{\circ} \mathrm{R}, 13^{\circ} \mathrm{L} \quad 0^{\circ} \mathrm{R}, 15^{\circ} \mathrm{L} \quad 0^{\circ} \mathrm{R}, 16^{\circ} \mathrm{L} 15^{\circ} \mathrm{R}, 36^{\circ} \mathrm{L}$

Similar results were given by a second, similar patient following removal of an acoustic neuroma.

(3) Positional nystagmus beating ageotropically with reduction of VOR by lateral acceleration (the eye movement produced by gravity is in the opposite direction to the acceleration vector; the eye movement produced by lateral acceleration is in the same direction as the vector). We have not yet found a patient showing this pattern.

(4) Positional nystagmus beating geotropically with reduction of the VOR by lateral acceleration in the head eccentric attitude (the eye movements provoked by gravity and lateral acceleration are both in the same direction as the acceleration vector).

Case example: A38668. Female aged 64 years who, in 1968, was shown to have a cystic lesion extending from the level of $\mathrm{Cl}$ to the floor of the 4th ventricle which was successfully treated with radiotherapy. In summer 1985 there was a recurrence of symptoms. When examined in April 1986 she had diplopia due to a left convergent strabismus. Abduction was restricted bilaterally, but saccades in all directions were of near normal velocity. There was gaze paretic nystagmus in all directions, with bidirectional rebound nystagmus. Pursuit was absent and there was "slow build up" optokinetic nystagmus. Vestibular ocular reflexes were hypoactive. Ocular counterrolling was intact. There was positional nystagmus beating leftwards when lying horizontally with the left ear down and beating rightwards with the right ear down. Magnetic resonance imaging demonstrated a low density mass, probably an ependymoma, which lay to the left of the midline, extending from the upper cervical cord to the floor of the IVth ventricle and invading the low brainstem and posterior vermis of the cerebellum. The gains of the slow phase eye movements evoked by head oscillation in the eccentric position were uniformly reduced by $18 \%$ at 0.1 and $1.2 \mathrm{~Hz}$ and by $30 \%$ at $0.5 \mathrm{~Hz}$. The slow phase eye movements provoked by the gravity acceleration vector in this patient were in the same direction as the slow phase eye movements provoked by the tangential acceleration vector during head eccentric oscillation.
(5) Positional nystagmus beating geotropically with enhancement of the VOR by lateral acceleration (the eye movement provoked by gravity is in the same direction as the acceleration vector while the eye movement provoked by lateral acceleration is in the opposite direction).

Case example: B11526. Male aged 50 years who, in 1980 , developed headaches, neck pain, unsteadiness and rotatory vertigo with vomiting. A CT scan demonstrated a posterior fossa mass. Craniotomy revealed a tumour which was seen protruding between the two cerebellar hemispheres in the midline, which biopsy showed to be a low grade astrocytoma. The tumour and lining of the cystic cavity were removed. In July 1986 the patient was reviewed. He complained only of "height vertigo" and dizziness on neck extension. On examination, pursuit was mildly hypometric, there was right beating positional nystagmus evoked with the right ear down, and a left beating positional nystagmus evoked with the left ear down (fig 4). There was some weakness of the shoulder girdle. A highresolution CT scan at this time (fig 5) showed a large CSF density space between the cerebellar hemispheres with no evidence of recurrence of the tumour. Slow phase eye movement responded to oscillation with head centred were normal. With head eccentric the gain of the slow phase eye movements was significantly increased at low frequencies of stimuli for head leftwards stimulation (figs $2 \mathrm{~b}, 3 \mathrm{c}$ ). The slow phase eye movements provoked by the gravity acceleration vector in this patient were in the opposite direction to the slow phase eye movements provoked by the tangential acceleration vector during head eccentric oscillation. Response phase data for leftwards (L) and rightwards $(R)$ slow phase eye movements were as follows:

$\begin{array}{lcccc} & 1.2 \mathrm{~Hz} & 0.5 \mathrm{~Hz} & 0.1 \mathrm{~Hz} & 0.02 \mathrm{~Hz} \\ \text { Head centre } & 6^{\circ} \mathrm{R}, 0^{\circ} \mathrm{L} & 4^{\circ} \mathrm{R}, 0^{\circ} \mathrm{L} & 5^{\circ} \mathrm{R}, 6^{\circ} \mathrm{L} & 9^{\circ} \mathrm{R}, 9^{\circ} \mathrm{L} \\ \text { Head eccentric } & -4^{\circ} \mathrm{R}, 9^{\circ} \mathrm{L} & 5^{\circ} \mathrm{R}, 5^{\circ} \mathrm{L} & 5^{\circ} \mathrm{R}, 2^{\circ} \mathrm{L} & 17^{\circ} \mathrm{R}, 6^{\circ} \mathrm{L}\end{array}$

In addition to the above, normal responses have been found in a variety of patients whom one might have expected, from their symptoms or signs, to have otolithic disorders. For example: (1) "Benign paroxysmal vertigo" attributed to degeneration of the otolith organ. Two patients with classical paroxysmal vertigo with torsional nystagmus provoked by positioning failed to show abnormal responses with head eccentric rotation.

(2) Cerebellar-pontine angle lesion from presumed ectatic basilar artery loop. A normal pattern of responses was found in this patient who had deafness and complete canal paresis on the left side. Perhaps of significance, this patient did not have positional or spontaneous nystagmus which may indicate that she compensated well for the loss of vestibular function. 


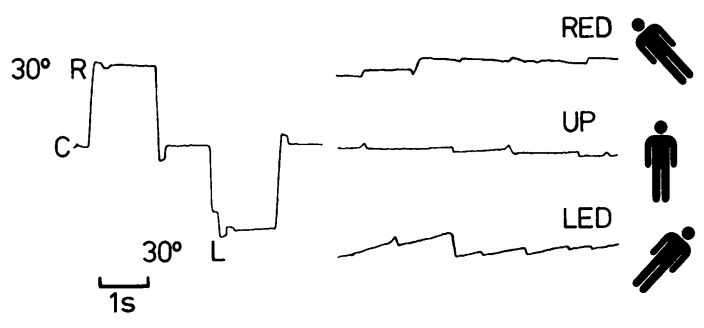

Fig 4 Patient B11526. Positional dark nystagmus with lateral tilts of $45^{\circ}$ left and right.

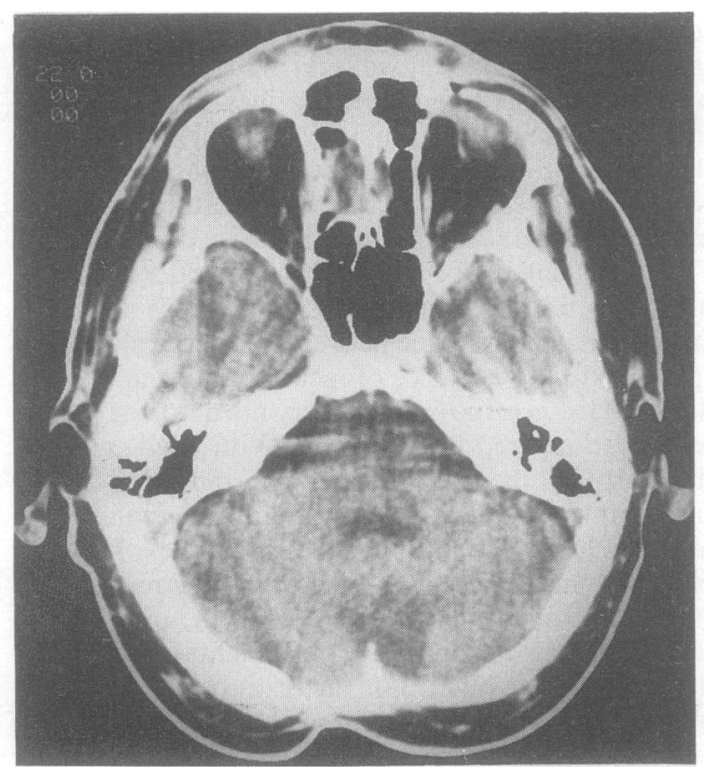

Fig 5 Patient B11526. High resolution CT scan showing a large CSF density space between the cerebellar hemispheres.

(3) Chronic vertigo of unknown aetiology. Normal responses were identified in a patient with a four year history of illusory sensations of linear motion of herself and the ground, "mal de debarquement". The linear nature of the illusory movement made one suspect a disorder of otolith function.

\section{Discussion}

The findings show that rotational vestibular testing performed with head eccentric oscillation can reveal abnormalities of eye movement responses in patients who are normal on conventional head centred rotational testing (for example, type v, patient B11526). Therefore, this method of investigating vestibular ocular reflexes has the potential of adding significantly to the understanding of a patient's neuro-otological status. However, to date, we have only detected abnormalities in the head eccentric responses in patients in whom there is also positional nystagmus. This suggests that the technique may be more of a research tool than a decisive clinical investigation for it constitutes an over-elaborate procedure in comparison with the simple task of identifying positional nystagmus!

The significant findings in our patients which relate to current concepts of otolith function are the enhanced gains (velocity ratios) associated specifically with high frequency stimuli (2, patient B45846) or low frequency stimuli (5, patient B11526).

(a) High frequency abnormalities In normals, high velocities of eye movement attributable to otolith stimulation occur particularly with high frequency stimuli. The patient who had had an acoustic neuroma removed showed higher gains for both centred and eccentric stimuli for movement in the direction of the intact side at the higher stimulus frequencies. Thus it is likely that her asymmetrical responses in the head eccentric position are attributable to otolithic effects which have become asymmetrical because of the nerve section, in a similar way to the canal reflexes. It is notable that in patients with acoustic neurinectomies, the eye movements evoked by movement to the lesioned side are similar for both head attitudes. This observation indicates that whereas their remaining canal could generate eye movements in both directions, the intact otolith on one side could only generate eye movements towards the contralateral side.

(b) Low frequency abnormalities The type 5 example of abnormal response (B11526) shows a unidirectionally enhanced velocity ratio at low stimulus frequencies. Responses provoked by low frequency stimuli may not be related to the otolith contribution to compensatory reflexes which our data suggest is mainly high frequency sensitive. Instead, they may reflect abnormalities of otolith function related to adaptation and eye velocity storage mechanisms, ${ }^{12-15}$ both of which involve long time constants which one would associate with low frequency performance.

In types 3 and 5 the slow phase lateral eye movements evoked by the gravity acceleration vector are in the opposite direction to the enhancement of the slow phase eye movements produced by lateral acceleration during oscillation with head eccentric. In types 2 and 4 the eye movements evoked by gravity are in the same direction to those produced by lateral acceleration during oscillation with head eccentric. These observations underline two unresolved problems of otolith pathophysiology. Firstly, if we are to consider ageotropic nystagmus as a "released" compensatory phenomenon, what is the explanation of geotropic nystagmus which is in the opposite direction? Sec- 
ondly, how can the response to phasic linear acceleration, even at low frequencies, be in the opposite direction to the static response as indicated by the direction of positional nystagmus?

One of our original intentions was to design a technique to investigate the otolith contribution to compensatory eye movements in patients with neurootological disease. In some, particularly those with feelings of "mal de debarquement", one suspects otolithic abnormalities because of the nature of their symptoms. However, not all such patients show abnormal responses on head eccentric testing although, clearly, the technique reveals some otolithoculomotor abnormalities. These observations indicate that this method can provide valuable insights into vestibular-oculomotor pathophysiology, and will be worth extending to motion about other axes such as head pitch. However the negative findings also underline the fact that we have to rely too heavily on vestibular-ocular performance as an indicator of the integrity of balance.

Dr AM Bronstein was supported by the Brain Research Trust.

\section{References}

1 Graybiel A. Measurement of otolith function in man. In: Kornhuber HH, ed. Vestibular System part 2: Psychophysics, Applied Aspects and General Interpretations. Handbook of sensory physiology, vol. VI. Berlin: Springer-verlag, 1974;233-66.

2 Page NGR, Gresty MA. Motorist's vestibular disorientation syndrome. J Neurol Neurosurg Psychiatry 1985;48:729-35.

3 Brain WR. On the rotated or "cerebellar" posture of the head. Brain 1926;49:61-75.

4 Halmagyi GM, Gresty MA, Gibson WPR. Ocular tilt reaction with peripheral vestibular lesion. Ann Neurol 1978;6:80-3.

5 Nylen CO. Positional nystagmus: a review and future prospects. J Laryngol Otol 1950;64:295-318.

6 Cawthorne T, Hinchcliffe R. Positional nystagmus of the central type as evidence of subtentorial metastases. Brain 1961;74:415-26.

7 Keane JR. Ocular skew deviation. Arch Neurol 1975;32:185-90

8 Keane JR. Alternating skew deviation: 47 patients. Neurology 1985;35:725-8.

9 Barnes GR. Vestibular mechanisms (review article). Clin Physics Physiol Meas 1979;1:3-40.

10 Gresty MA, Bronstein AM. Otolith stimulation evokes compensatory reflex eye movements of high velocity when linear motion of the head is combined with concurrent angular motion. Neurosci Lett 1986;65: 149-54.

11 Gresty MA, Bronstein AM, Barratt H. Eye movement responses to combined linear and angular head movement. Exp Brain Res 1987;65:377-84.

12 Benson AJ. Modification of the Response to Angular Accelerations by Linear Accelerations. In: Kornhuber HH, ed Vestibular System Part 2: Psychophysics, Applied Aspects and General Interpretations. Handbook of Sensory Physiology, vol VI. Berlin, Springer-verlag 1974:281-320.

13 Guedry FE Jr. Orientation of the rotation-axis relative to gravity: its influence on nystagmus and the sensation of rotation. Acta Otolaryng (Stockh) 1965;60: $30-48$.

14 Schrader V, Koenig E, Dichgans J. The effect of lateral head tilt on horizontal postrotatory nystagmus I and II and the Purkinje effect. Acta Otolaryngol (Stockh) 1985;100:98-105.

15 Gresty M, Barratt H, Bronstein A, Page N. Clinical aspects of otolith-oculomotor relationships. In: Keller EL, Zee DS, eds. Adaptive Processes in Visual Oculomotor Systems. Oxford: Pergamon Press, 1986:357-66. 